\title{
Association between Loss of Skeletal Muscle Mass and Mortality and Tumor Recurrence in Hepatocellular Carcinoma: A Systematic Review and Meta-Analysis
}

\author{
Ke-Vin Chang ${ }^{a-c}$ Jin-De Chen ${ }^{b, d}$ Wei-Ting Wu ${ }^{a}$ Kuo-Chin Huang ${ }^{b}$ e \\ Chia-Tzu Hsu ${ }^{b}$ Der-Sheng Han ${ }^{a-c, f}$ \\ ${ }^{a}$ Department of Physical Medicine and Rehabilitation and ${ }^{b}$ Community and Geriatric \\ Medicine Research Center, National Taiwan University Hospital, Bei-Hu Branch, \\ ${ }^{c}$ Department of Physical Medicine and Rehabilitation, National Taiwan University College of \\ Medicine, dDepartment of Gastroenterology, National Taiwan University Hospital, Bei-Hu \\ Branch, e Department of Family Medicine, National Taiwan University College of Medicine, \\ and ${ }^{f}$ Health Science and Wellness Center, National Taiwan University, Taipei, Taiwan, ROC
}

\section{Keywords}

Cancer $\cdot$ Hepatocellular carcinoma $\cdot$ Muscle mass $\cdot$ Sarcopenia $\cdot$ Survival

\begin{abstract}
Background: Hepatocellular carcinoma (HCC) has multiple prognostic factors, and there is an increase in knowledge about the body composition and physical status of patients with HCC. The present meta-analysis aimed to explore whether loss of skeletal muscle mass is associated with mortality and tumor recurrence in patients with HCC. Method: A systematic search was conducted for published literature using PubMed, Embase, and Scopus. We included cohort or case-control studies investigating patients with HCC. The primary and secondary outcomes were the associations of loss of skeletal muscle mass with overall survival and tumor recurrence, respectively, expressed by a summary hazard ratio (HR) and $95 \%$ confidence interval (CI). Result: A total of 13 studies comprising 3,111 patients were included. The summary HRs calculated by either univariate or multivariate analysis both suggested a significant association between sarcopenia and all-cause mortality (crude HR $=2.04,95 \%$ CI: 1.74-2.38; adjusted HR $=1.95,95 \% \mathrm{CI}: 1.60-2.37$ ). Similarly, loss of skeletal muscle mass was associated with tumor recurrence (crude HR $=1.85,95 \%$ CI: 1.44-2.37; adjusted HR $=1.76,95 \%$ CI: $1.27-$ 2.45). The stratified analysis showed that treatment types and inclusion of body mass index or body weight in the Cox regression model did not modify both clinical outcomes. With an increase in cut-off values of muscle mass on computed tomography images (especially for male patients), there was an insignificant trend of stronger associations between loss of skel-
\end{abstract}


etal muscle mass and all-cause mortality. Conclusion: Loss of skeletal muscle mass is associated with increased all-cause mortality and tumor recurrence in patients with HCC. Further prospective studies incorporating measurements of muscle strength and physical function are warranted to see whether inclusion of both parameters better predicts the outcome than use of muscle mass only.

(c) 2017 S. Karger AG, Basel

\section{Introduction}

Primary liver cancer is the sixth most prevalent cancer globally, and hepatocellular carcinoma (HCC) accounts for $80 \%$ of the cases, followed by intrahepatic cholangiocarcinoma [1]. HCC has the third highest mortality rate worldwide, with the 5-year survival rate ranging from 10 to $28 \%$ $[2,3]$. Multiple factors are associated with clinical outcomes of HCC, including liver function, tumor site, treatment type, and comorbidity [4,5]. Early detection and referral for curative treatments such as surgical resection and radiofrequency ablation are the most effective approaches to increase the survival of patients. Maintenance of nutritional balance and physical capability is another key element in improving the prognosis in the late cancer stage. In recent years, body composition parameters such as muscle mass and visceral adiposity have been widely investigated for possible roles in influencing clinical outcomes of patients with HCC.

Sarcopenia, defined as a decrease in muscle mass and strength, is an important cause of functional decline and is associated with aging and chronic illness [6]. Multinutrient supplementation as well as resistive strengthening exercise is effective to reverse sarcopenia and subsequent adverse health outcomes [7, 8]. Many researchers are currently investigating the relationship between loss of skeletal muscle mass and cancer prognosis [9]. Therefore, the present meta-analysis aimed to explore whether loss of skeletal muscle mass is associated with mortality and tumor recurrence in patients with HCC.

\section{Materials and Methods}

Search Strategy

All literature published until September 2017 was searched using three electronic databases, PubMed, Embase, and Scopus, without language restriction. The reference lists of the retrieved articles and relevant systematic review and meta-analyses were also scrutinized for eligible studies. The following key terms were used for the literature search: ["sarcopenia" or "fragility" or "skeletal muscle"] and ["hepatocellular carcinoma" or "hepatic malignancy" or "liver tumor"] (online suppl. Appendix 1; for all online suppl. material, see www.karger.com/doi/10.1159/000484950).

\section{Study Selection}

The retrieved studies were eligible for qualitative and quantitative analysis if they (1) were cohort studies, case-control studies, and follow-ups of clinical trials; (2) investigated patients with HCC; (3) provided measurement of skeletal muscle mass or function; and (4) reported data related to overall survival or recurrence-free survival. The studies were excluded if they (1) probed participants with malignancy other than HCC; (2) only recruited patients with liver cirrhosis but without concomitant liver cancers; and (3) lacked definition of skeletal muscle mass loss for their target population and measurement of prognostic factors related to cancer recurrence or mortality.

Data Extraction and Quality Assessment

Two authors independently extracted the data from the included studies and assessed their quality by using the Newcastle-Ottawa Scale [10-12]. The following details were presented in this review: first author's name, year of publication, patients' characteristics, enrolled numbers, sex ratio, muscle mass measurement, definition of skeletal muscle mass loss, subsequent treatment for hepatic malignancy, outcome variables, and 
Chang et al.: Association between Skeletal Muscle Mass Loss and Hepatocellular Carcinoma

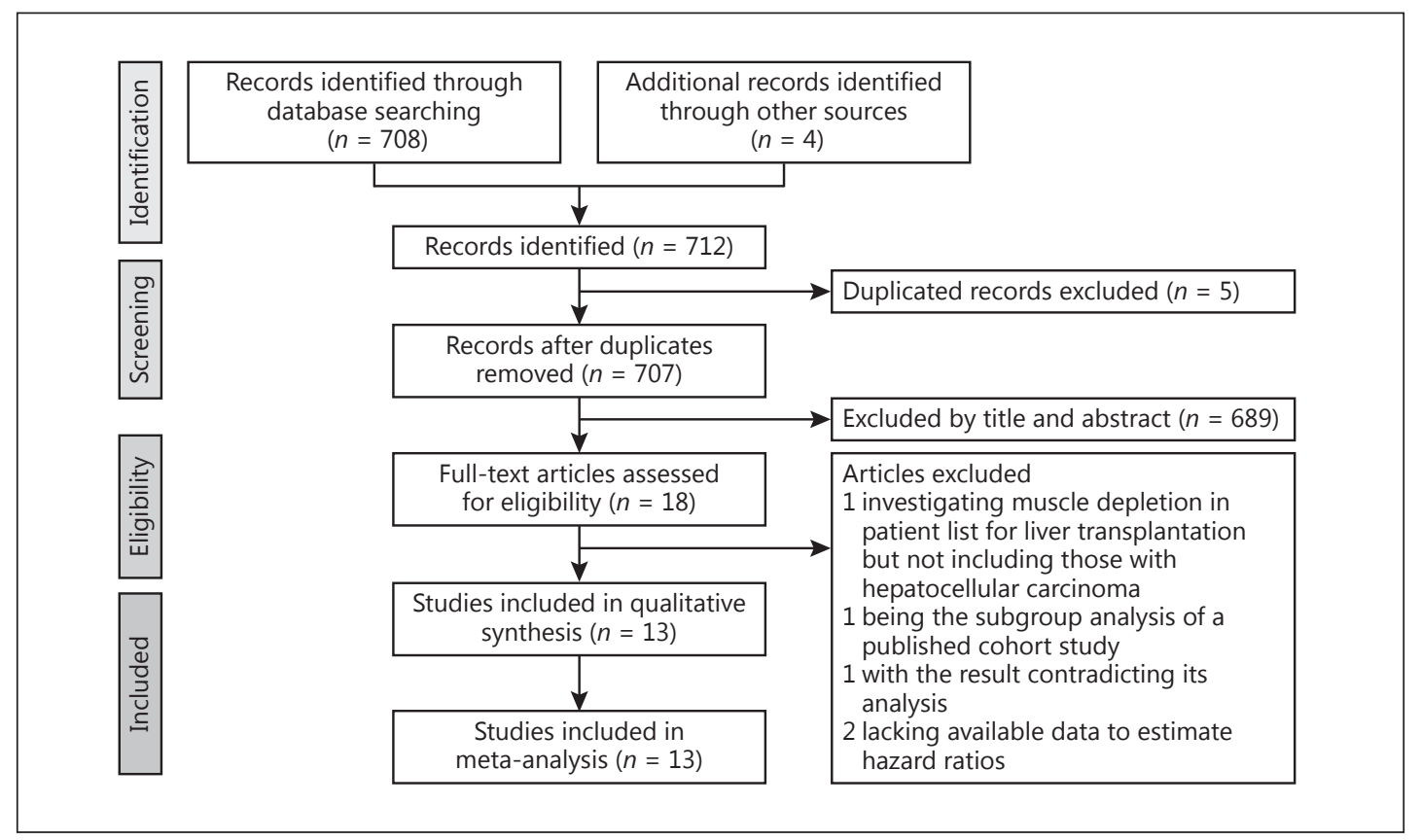

Fig. 1. Preferred Reporting Items for Systematic Reviews and Meta-Analyses (PRISMA) flow diagram for the study selection process.

adjusted major confounders. The Newcastle-Ottawa Scale was used to evaluate the quality of nonrandomized trials, encompassing three major aspects: (1) selection (representativeness of the exposed cohort, selection of the nonexposed cohort, ascertainment of exposure, and demonstration of outcome of interest), (2) comparability of the cohort, and (3) outcome assessment and duration and adequacy of follow-up. Any discrepancy between both reviewers was resolved by discussion and consensus.

\section{Statistical Analysis}

The primary and secondary outcomes were the associations of skeletal muscle mass loss with the overall survival and recurrence-free survival, respectively, expressed by crude and adjusted hazard ratios (HRs). Most of the HRs were derived from univariate and multivariate Cox regression analyses. Some of them were calculated from the numbers at risk shown on the Kaplan-Meier survival plot.

As the severity of included participants might vary across studies, a random effect model was used to calculate the summary HRs, along with a 95\% confidence interval (CI) for the association between sarcopenia and overall mortality and tumor recurrence [13]. A sensitivity analysis was performed by eliminating studies that included patients with primary liver malignancy other than HCC. A stratified analysis for the adjusted HRs of the remaining studies that included only patients with HCC was conducted according to difference groups of treatment options and body mass index. The Cochrane $Q$ test and $I^{2}$ statistics were employed to explore between-study heterogeneity, and an $I^{2}>50 \%$ was considered statistically significant. The Egger test and funnel plots were used to detect potential publication bias [14]. All the calculations were performed using Comprehensive Meta-Analysis Software version 3 (Biostat, Englewood, NJ, USA), with $p<0.05$ considered to be statistically significant.

\section{Results}

\section{Search Result}

Of the 708 records that were identified in the literature search, 18 full-text articles were assessed. We further excluded 5 studies: 1 investigated muscle depletion in patients on the 
Chang et al.: Association between Skeletal Muscle Mass Loss and Hepatocellular Carcinoma

\begin{tabular}{|c|c|c|c|c|c|c|c|}
\hline 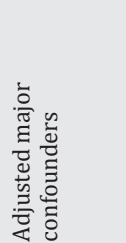 & 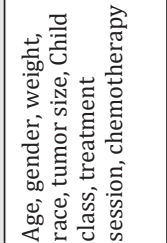 & 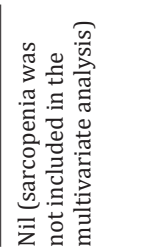 & 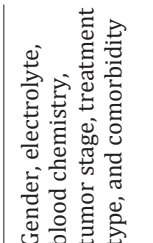 & 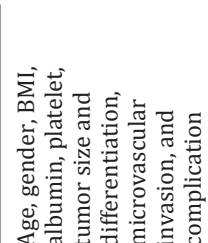 & 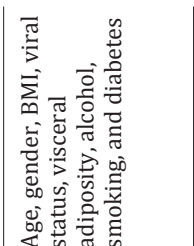 & 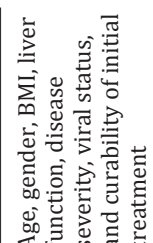 & 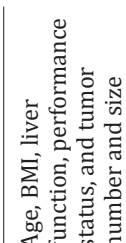 \\
\hline 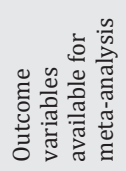 & 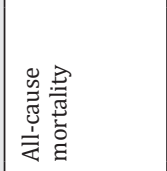 & 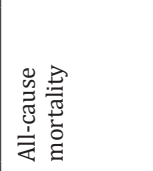 & 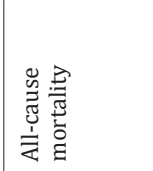 & 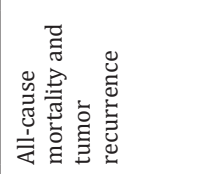 & 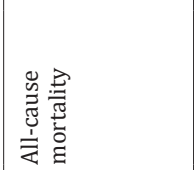 & 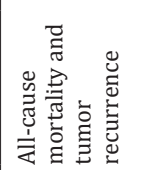 & 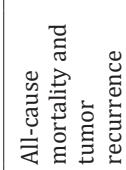 \\
\hline 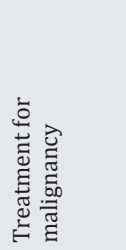 & 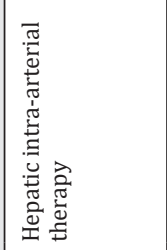 & 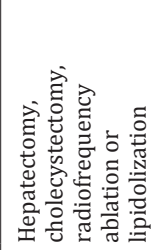 & 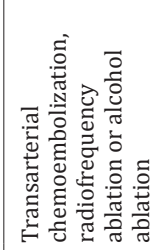 & 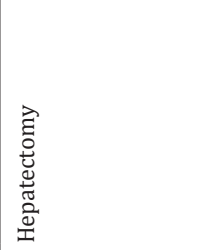 & 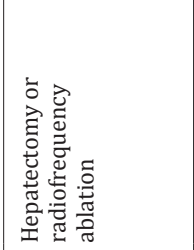 & 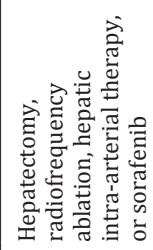 & 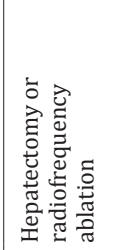 \\
\hline 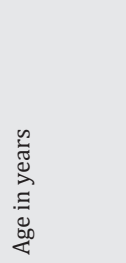 & 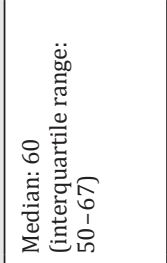 & 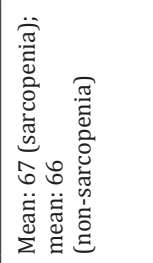 & 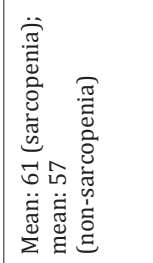 & 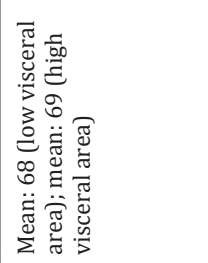 & 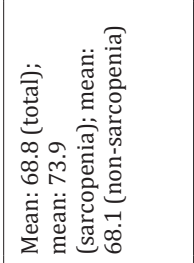 & & 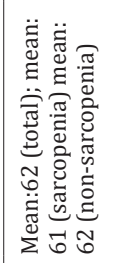 \\
\hline 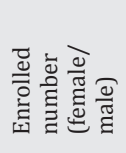 & 竞 & 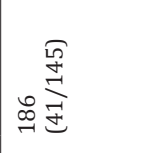 & 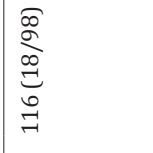 & 勇 & 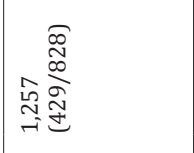 & $\approx \sum_{0}^{0}$ & 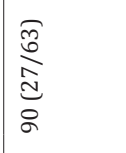 \\
\hline 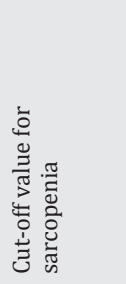 & 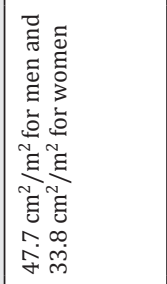 & 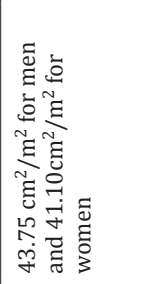 & 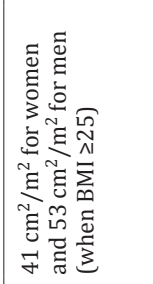 & 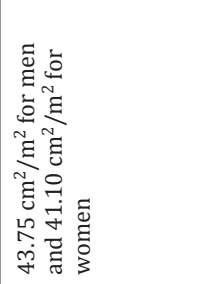 & 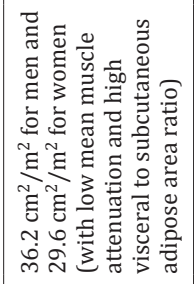 & 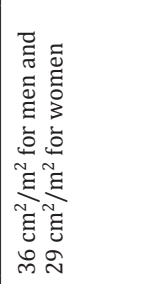 & 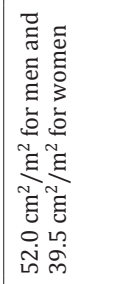 \\
\hline 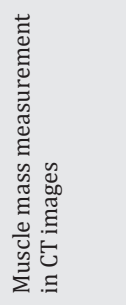 & 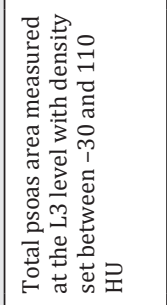 & 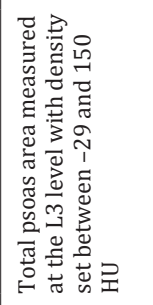 & 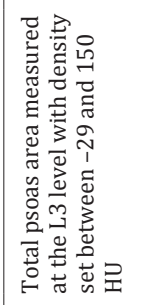 & 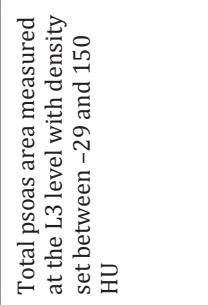 & 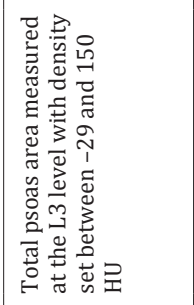 & 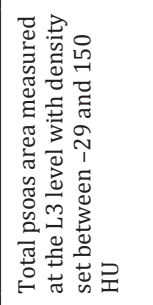 & 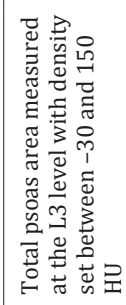 \\
\hline 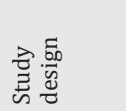 & 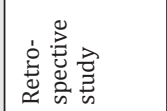 & 它总总 & 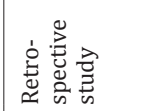 & 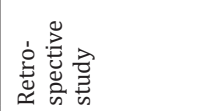 & 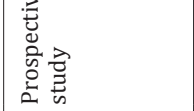 & 它总总 & 它总离 \\
\hline 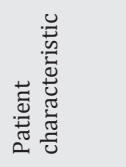 & 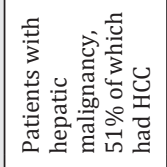 & 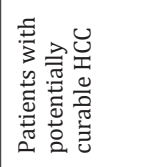 & 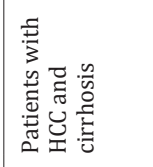 & 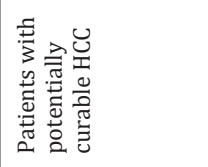 & 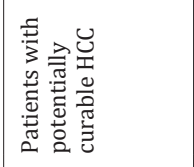 & 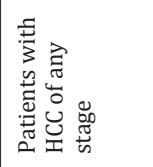 & 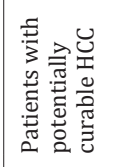 \\
\hline 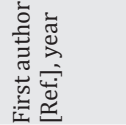 & 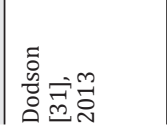 & 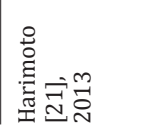 & 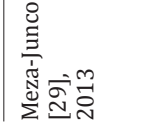 & 폴로요 & 营 & 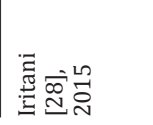 & 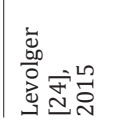 \\
\hline
\end{tabular}




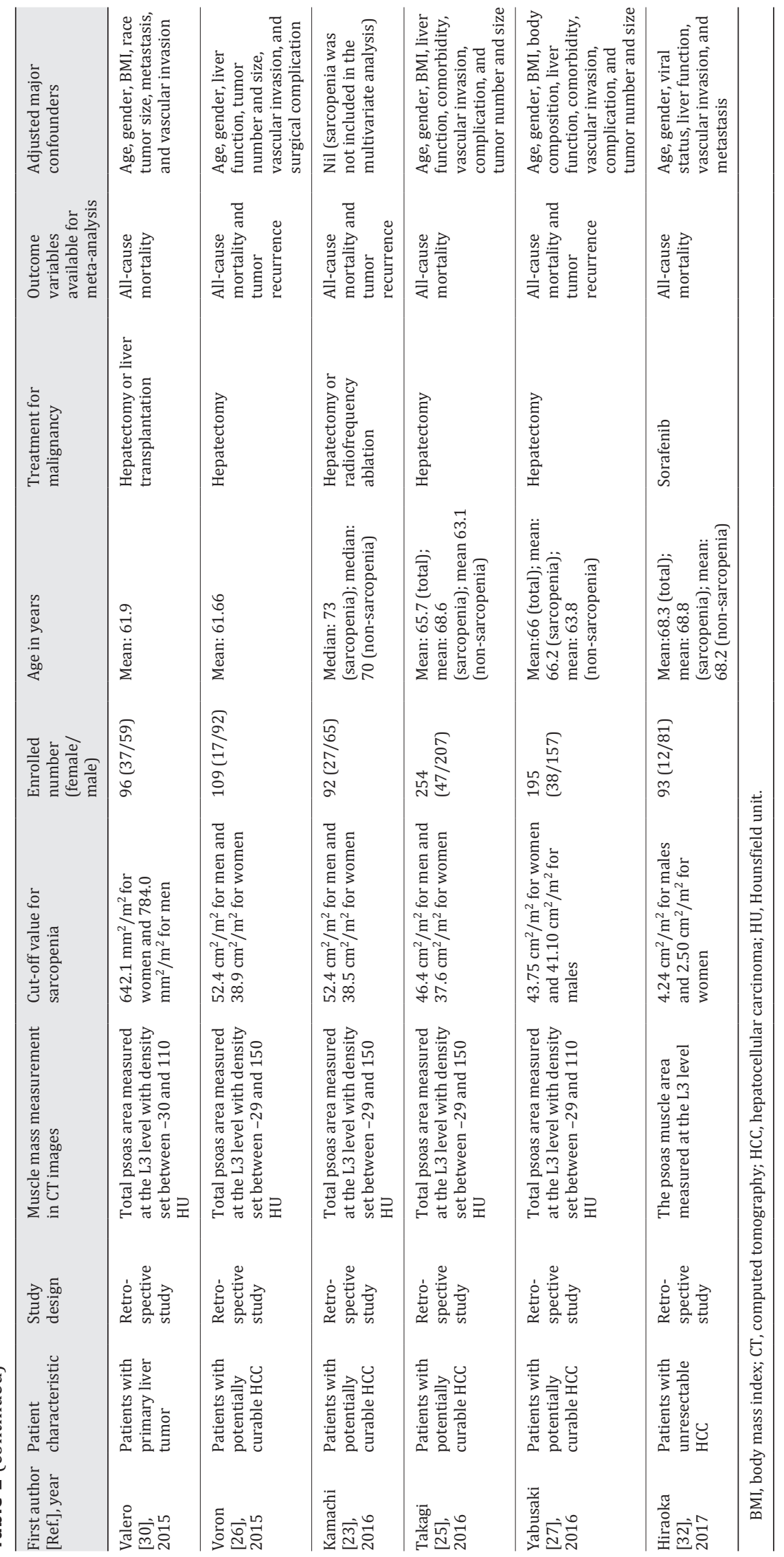


Chang et al.: Association between Skeletal Muscle Mass Loss and Hepatocellular Carcinoma

Table 2. Quality assessment by using the Newcastle-Ottawa Scale for the included studies

\begin{tabular}{|c|c|c|c|c|c|c|c|c|c|}
\hline $\begin{array}{l}\text { First author [Ref.], } \\
\text { year }\end{array}$ & $\begin{array}{l}\text { Representative } \\
\text { of sarcopenia } \\
\text { patients }\end{array}$ & $\begin{array}{l}\text { Selection } \\
\text { of } \\
\text { control }\end{array}$ & $\begin{array}{l}\text { Ascertain of } \\
\text { sarcopenia } \\
\text { measurement }\end{array}$ & $\begin{array}{l}\text { Outcome of } \\
\text { interest not } \\
\text { present at start }\end{array}$ & $\begin{array}{l}\text { Compara- } \\
\text { bility } \\
\text { of cohorts }\end{array}$ & $\begin{array}{l}\text { Assessment } \\
\text { of outcome }\end{array}$ & $\begin{array}{l}\text { Enough } \\
\text { follow-up } \\
\text { period }\end{array}$ & $\begin{array}{l}\text { Adequacy } \\
\text { of follow-up }\end{array}$ & $\begin{array}{l}\text { Total } \\
\text { points }\end{array}$ \\
\hline Dodson [31], 2013 & $*$ & $*$ & * & $*$ & $* *$ & $*$ & $*$ & $*$ & 9 \\
\hline Harimoto [21], 2013 & * & * & $*$ & $*$ & $*$ & $*$ & * & $*$ & 8 \\
\hline Meza-Junco [29], 2013 & * & $*$ & * & $*$ & ** & $*$ & - & $*$ & 8 \\
\hline Itoh [22], 2014 & * & $*$ & * & * & $* *$ & * & $*$ & $*$ & 9 \\
\hline Fujiwara [20], 2015 & $*$ & $*$ & * & $*$ & $* *$ & $*$ & $*$ & $*$ & 9 \\
\hline Iritani [28], 2015 & * & $*$ & * & $*$ & $* *$ & $*$ & $*$ & $*$ & 9 \\
\hline Levolger [24], 2015 & * & * & $*$ & $*$ & ** & $*$ & $*$ & $*$ & 9 \\
\hline Valero [30], 2015 & * & $*$ & * & * & $* *$ & * & $*$ & $*$ & 9 \\
\hline Voron [26], 2015 & $*$ & $*$ & $*$ & $*$ & $* *$ & $*$ & $*$ & $*$ & 9 \\
\hline Kamachi [23], 2016 & $*$ & * & * & $*$ & $*$ & $*$ & $*$ & $*$ & 8 \\
\hline Takagi [25], 2016 & * & * & $*$ & $*$ & $* *$ & * & $*$ & $*$ & 9 \\
\hline Yabusaki [27], 2016 & $*$ & $*$ & * & * & $* *$ & * & - & - & 7 \\
\hline Hiraoka [32], 2017 & * & $*$ & $*$ & $*$ & $* *$ & * & $*$ & $*$ & 9 \\
\hline
\end{tabular}

waiting list for liver transplantation but excluded those with HCC [15], 1 was the subgroup analysis of a published cohort study [16], 1 presented with a result inconsistent with its statistical analysis [17], and 2 lacked available data to estimate HRs of the association between sarcopenia and our desired outcomes $[18,19]$. The final meta-analysis included 13 studies, comprising 3,111 patients (Fig. 1).

\section{Study Characteristics}

Regarding the research design, 12 studies were conducted by retrospectively reviewing the computed tomography (CT) scans and medical records from a preexisting patient cohort, whereas 1 consecutively enrolled patients based on a predesignated protocol [20]. In terms of patient demographic characteristics, female patients accounted for $28.4 \%$ of the investigated population and the mean (or median) age of the enrolled patients in each study (or its subgroup) ranged between 57 and 73.9 years. Regarding the pattern of underlying malignancy, 7 studies included patients with resectable HCC [21-27], 3 included patients with any clinical stage of HCC $[20,28,29], 1$ included patients with primary liver tumors $(69.8 \%$ had HCC) [30], 1 included patients with hepatic cancers (50.5\% had HCC) undergoing intraarterial therapy [31], and 1 included patients with HCC following sorafenib treatment [32]. The muscle volume was estimated on the transverse CT image at the third lumbar vertebra level in all the selected studies, although the intensity window - (shown by using the Hounsfield scale) for recognizing skeletal muscles and cut-off points for sarcopenia - varied across trials (Table 1). The evaluation of the quality of the included studies is presented in Table 2.

\section{Overall Survival}

Not all the included studies reported HRs of overall survival or available data to compute them. Availability of both outcome measurements is detailed in Table 1 . The crude pooled HR of sarcopenia for overall survival from 11 included studies was 2.04 (95\% CI: 1.74-2.38) [20-22, 24-26, 28-32], while the adjusted summary HR from 10 selected trials was 1.95 (95\% CI: 1.60-2.37) (Fig. 2) [20, 22-26, 29-32]. The test for heterogeneity was not significant for both pooled values $\left(I^{2}<0.001\right.$ and $p$ value $=0.716$ for the crude HRs; $I^{2}=10.219$ and $p$ value $=0.349$ for the adjusted HRs). Potential publication bias was detected regarding the adjusted HRs ( $p$ value $=0.016$ for the Egger's test) but not for crude HRs ( $p$ value $=0.100$ for the Egger's test). The funnel plot of the above-mentioned variables is shown in online suppl. Appendix II. We also imputed the potential unpublished studies in the funnel plot regarding adjusted HRs (by using the function of trim and fill of the statistical software). The pooled HR 
Chang et al.: Association between Skeletal Muscle Mass Loss and Hepatocellular Carcinoma

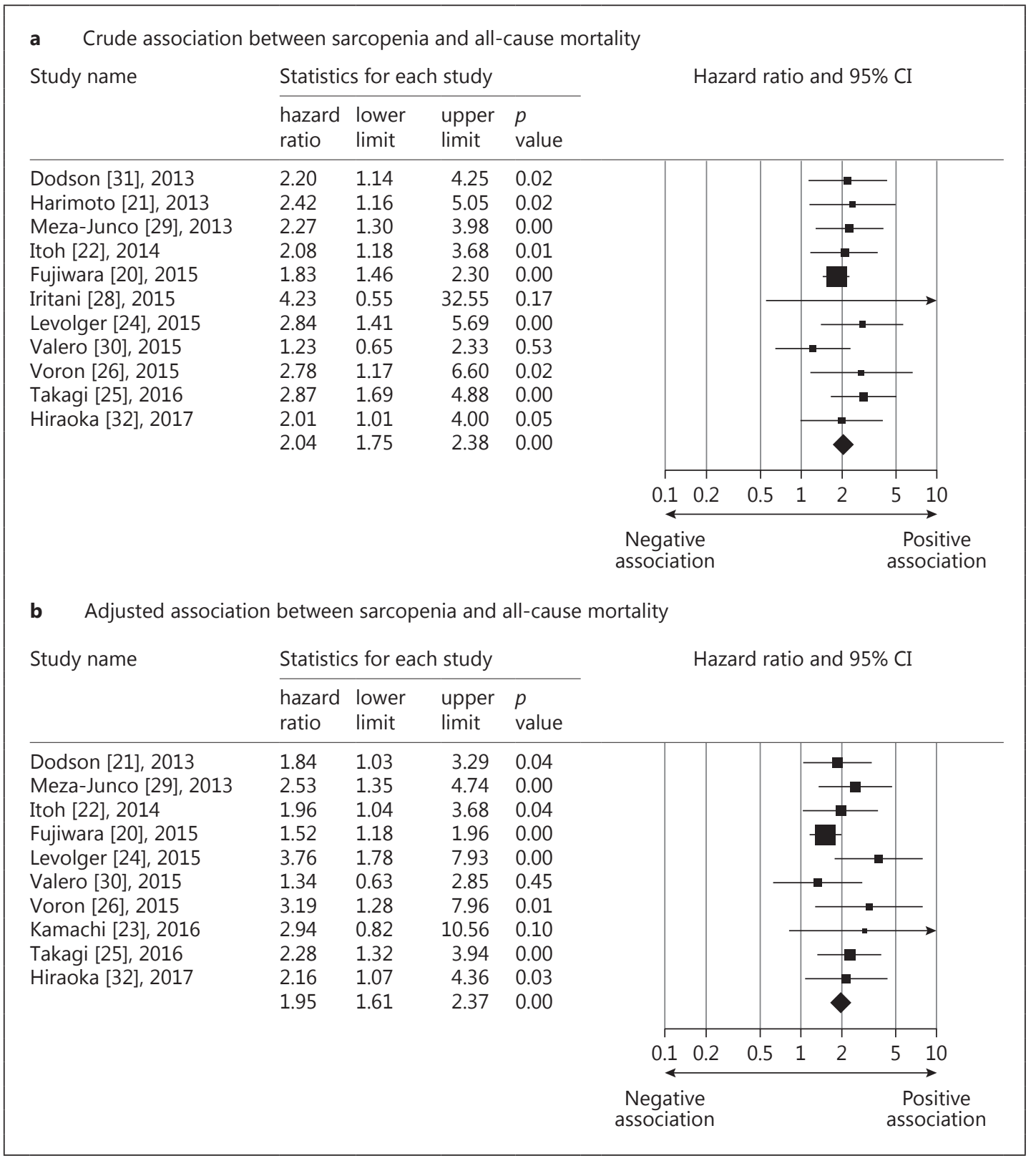

Fig. 2. Forest plot of the crude (a) and adjusted (b) associations between sarcopenia and all-cause mortality in patients with hepatocellular carcinoma.

decreased from 1.95 (95\% CI: 1.60-2.37) to 1.66 (95\% CI: 1.33-2.07) (online suppl. Appendix III). A sensitivity analysis was performed by eliminating 2 articles that included patients with primary liver malignancy other than HCC [30,31]. The crude and adjusted HRs became 2.09 (95\% CI: 1.78-2.47) and 2.11 (95\% CI: 1.66-2.69), respectively.

We further performed a stratified analysis for the adjusted HRs of the remaining studies that included only patients with HCC. We did not find the subsequent treatment (curative therapy vs. palliative treatment) to be a significant effect modifier for the association between loss of skeletal muscle mass and overall survival. The curative therapy included hepatectomy, radiofrequency ablation, and liver transplantation, whereas the palliative treatment 
Chang et al.: Association between Skeletal Muscle Mass Loss and Hepatocellular Carcinoma

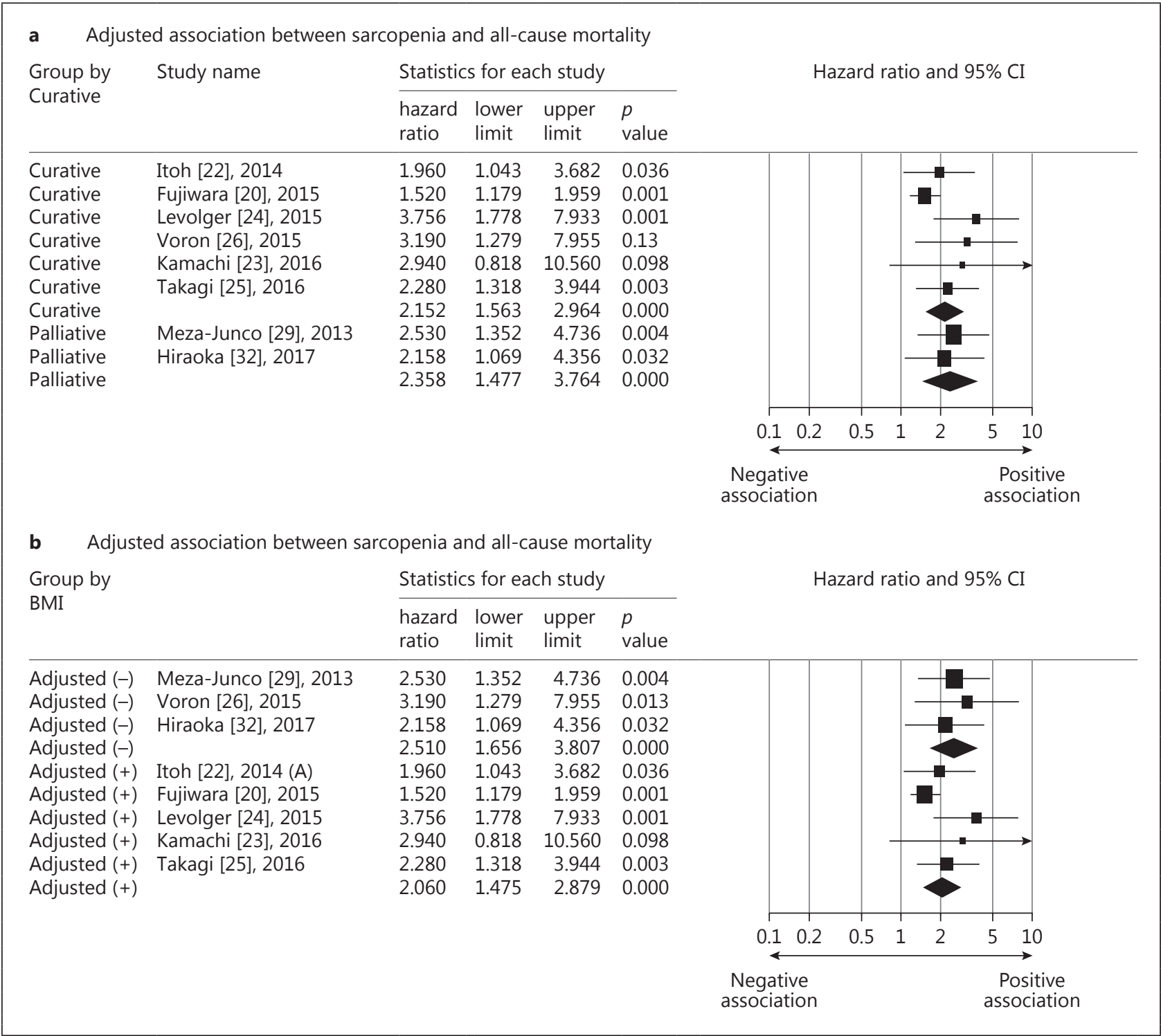

Fig. 3. Forest plot of the stratified analysis for the adjusted association between sarcopenia and all-cause mortality based on treatment type (a) and inclusion of body mass index (BMI) or weight (b) in the multivariate analysis.

comprised intraarterial chemoembolization and systemic chemotherapy. Another stratified analysis was conducted based on whether body mass index or body weight was included in the multivariate Cox regression models. We found that the summary HRs appeared similar in both groups with and without adjustment for body mass index (Fig. 3). Because the cut-off points for skeletal muscle mass loss varied among studies, we categorized them into three groups: group 1: $36.0-36.2 \mathrm{~cm}^{2} / \mathrm{m}^{2}$ for men, $29.0-29.6 \mathrm{~cm}^{2} / \mathrm{m}^{2}$ for women; group 2: $43-46.4$ $\mathrm{cm}^{2} / \mathrm{m}^{2}$ for men, 37.6-41.1 $\mathrm{cm}^{2} / \mathrm{m}^{2}$ for women; and group 3: $52.0-52.4 \mathrm{~m}^{2} / \mathrm{m}^{2}$ for men, 38.5$39.5 \mathrm{~cm}^{2} / \mathrm{m}^{2}$ for women. With the increase in the threshold values for skeletal muscle mass loss, the associations between sarcopenia and overall mortality became stronger, although the trend was not statistically significant (Fig. 4). 
Chang et al.: Association between Skeletal Muscle Mass Loss and Hepatocellular Carcinoma

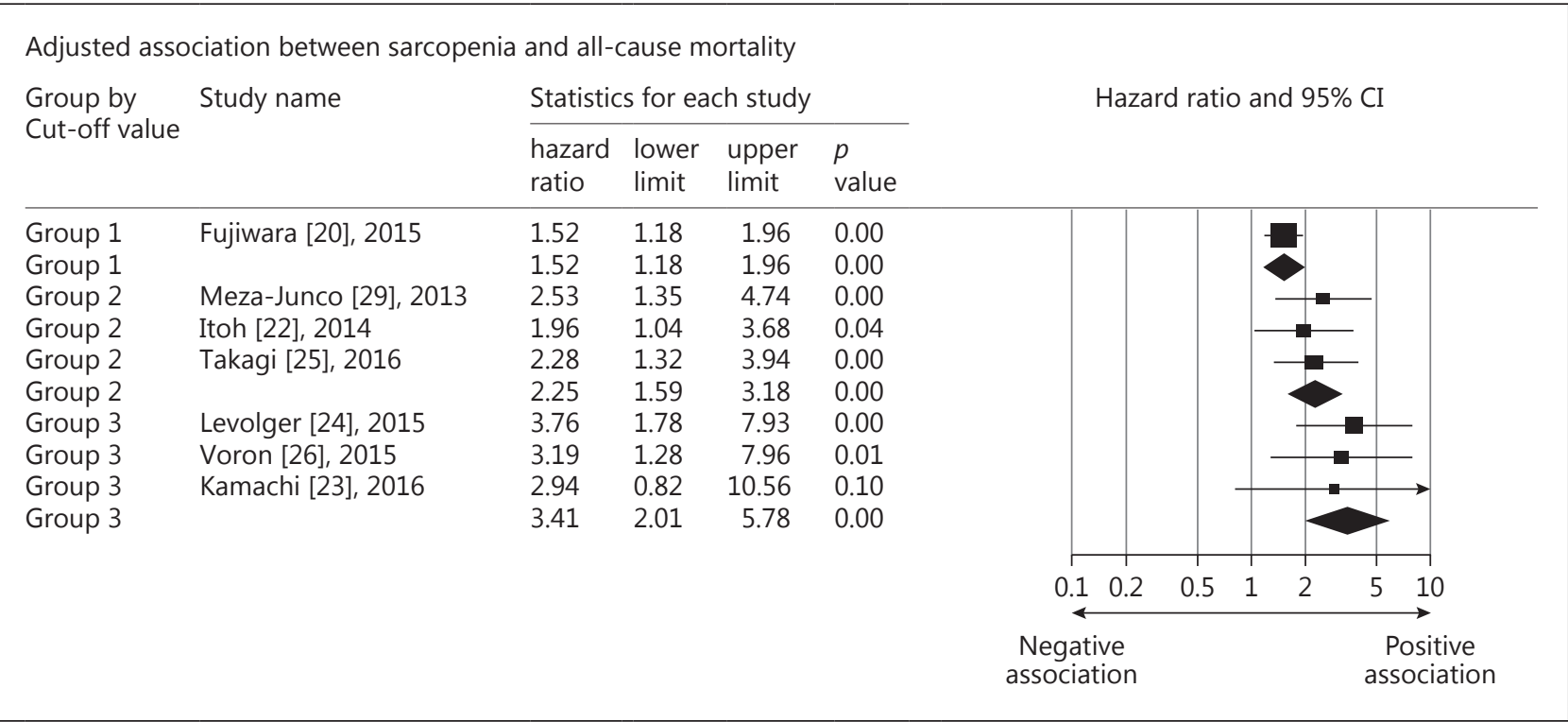

Fig. 4. Forest plot of the stratified analysis for the adjusted association between sarcopenia and all-cause mortality based on cut-off values of muscle cross-sectional area on computed tomography images (group 1: $36.0-36.2 \mathrm{~cm}^{2} / \mathrm{m}^{2}$ for men, $29.0-29.6 \mathrm{~cm}^{2} / \mathrm{m}^{2}$ for women; group $2: 43-46.4 \mathrm{~cm}^{2} / \mathrm{m}^{2}$ for men, $37.6-41.1 \mathrm{~cm}^{2} /$ $\mathrm{m}^{2}$ for women; group $3: 52.0-52.4 \mathrm{~m}^{2} / \mathrm{m}^{2}$ for men, $38.5-39.5 \mathrm{~cm}^{2} / \mathrm{m}^{2}$ for women).

\section{Recurrence-Free Survival}

Likewise, not all the included studies reported crude and adjusted HRs of tumor recurrence or had available data to estimate them (refer to Table 1 for availability of both outcome variables). The crude pooled HR of skeletal muscle mass loss for recurrence-free survival from 6 studies was calculated to be 1.85 (95\% CI: 1.44-2.37) [21, 22, 24, 26-28], while the adjusted summary HR from 4 selected trials was 1.76 (95\% CI: 1.27-2.45) (Fig. 5) [22, 23, 26, 27]. The test for heterogeneity was not significant for both pooled values $\left(I^{2}<0.001\right.$ and $p$ value $=0.577$ for the crude HRs; $I^{2}=43.85$ and $p$ value $=0.148$ for the adjusted HRs). No potential publication bias was detected regarding the crude ( $p$ value $=0.602$ for the Egger's test) and adjusted HRs ( $p$ value $=0.250$ for the Egger's test). The funnel plot of abovementioned variables was shown in online supplementary Appendix II. The stratified analysis was not performed owing to the small number of studies reporting on tumor recurrence.

\section{Discussion}

The relationship between loss of skeletal muscle mass and survival in patients with HCC was systematically assessed in this meta-analysis. Our study showed that sarcopenia was associated with an increase in all-cause mortality and the association remained after adjustment of confounders. Furthermore, patients with sarcopenia had a higher risk of tumor recurrence, consistently reflected by both crude and adjusted HRs. Additionally, the magnitude of association between skeletal muscle mass loss and all-cause mortality might change at the different cut-off values for skeletal muscle volume.

Sarcopenia is associated with many adverse outcomes including impaired cognition, depression, risks of fall, higher incidence of hospitalization, and mortality $[10,11,33,34]$. In 
Chang et al.: Association between Skeletal Muscle Mass Loss and Hepatocellular Carcinoma

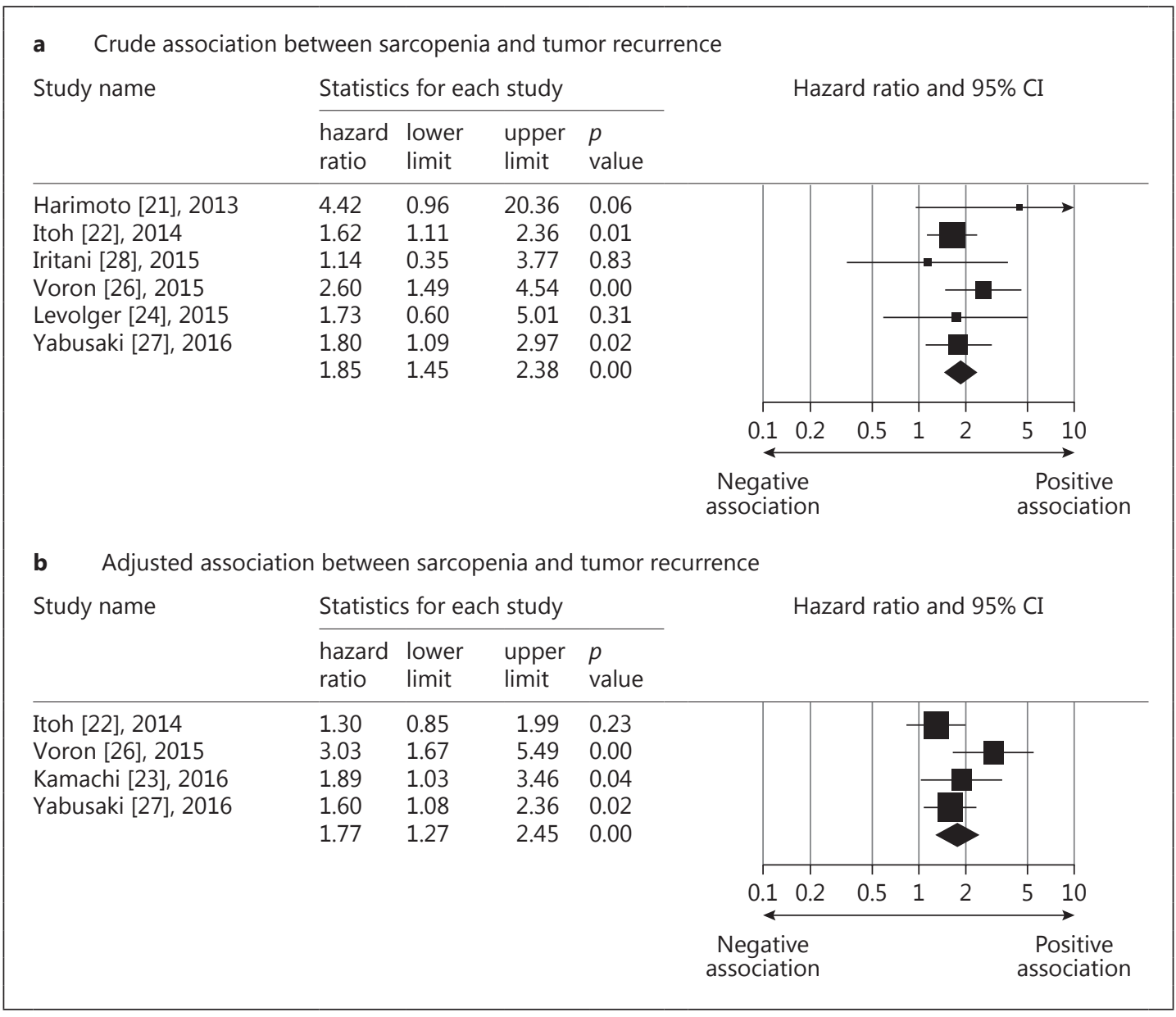

Fig. 5. Forest plot of the crude (a) and adjusted (b) associations between sarcopenia and mortality and tumor recurrence in patients with hepatocellular carcinoma.

2016, a meta-analysis of 10 studies demonstrated how patients with sarcopenia had an increased rate of mortality [35]. The target population of the study was miscellaneous, comprising community-dwelling residents and patients (1) after cystectomy, (2) being hospitalized due to acute illness, (3) diagnosed with pancreatic cancer, and (4) diagnosed with breast cancer. Another meta-analysis based on 38 studies demonstrated how a lower skeletal muscle index in adults was associated with poor overall survival [9]. Furthermore, this study investigated various kinds of malignancies and found that the association between sarcopenia and mortality might vary among different tumor types and across disease severity. Recently, there has been an increase in the number of studies investigating the prognostic factors in patients with HCC. We felt it crucial to separately summarize and analyze the association between loss of skeletal muscle mass and survival in patients with HCC as well as possible confounding factors.

Incorporating the latest evidence published in 2017, our meta-analysis showed that loss of skeletal muscle mass was associated with an increase in all-cause mortality in patients with HCC. The association was also independent of other prognostic factors such as age, sex, tumor size, cancer stage, and liver function. The pathogenesis of increased mortality in patients with sarcopenia remains uncertain. A commonly postulated mechanism is related to the decreased 


\section{Liver

Chang et al.: Association between Skeletal Muscle Mass Loss and Hepatocellular Carcinoma

secretion of certain cytokines such as insulin-like growth factor (IGF)-1 from reduced skeletal muscle mass. IGF-1 plays an important role in the growth of various organs, and its decrease in serum was significantly correlated with advanced clinicopathological parameters of HCC $[36,37]$. Sarcopenia is also linked to several proteolytic cascades such as the tumor necrosis factor (TNF)- $\alpha$ system [38]. TNF- $\alpha$ may promote tumor migration and invasion and is related to metastasis in HCC [38]. Nevertheless, it is unclear whether the increase in mortality may be a consequence of cancer-related cachexia rather than sarcopenia. Although skeletal muscle mass loss is a feature of cachexia, patients with cachexia tend to lose their weight more than those with sarcopenia [39]. Therefore, we separately analyzed adjusted HRs of all-cause mortality in the groups with and without adjustment of body mass index or body weight and found that the summary HRs appeared similar in both groups. Based on this finding, we believe that an independent association existed between loss of skeletal muscle mass and overall survival and was not merely a subcomponent of cachexia.

Hepatic resection and radiofrequency ablation are used as curative management for patients with early HCC. Our study revealed that loss of skeletal muscle mass was associated with an increased risk of tumor recurrence after curative therapy. The prognostic factors of tumor recurrence include age, sex, tumor size, histological grades, vascular invasion, and the stage of the background liver condition (cirrhosis) [5]. Similar to the relationship between sarcopenia and all-cause mortality, the mechanism for a higher recurrence rate in the sarcopenia group remains poorly understood. We believe that dysregulation of the cytokine system must play a vital role, which leads to carcinogenesis and distant metastasis. Another speculation is that sarcopenia might be an adverse consequence of liver cirrhosis, which results in an imbalance between protein synthesis and degradation [40]. Liver cirrhosis was found to be an independent factor of late recurrence, which is mainly caused by second primary lesions due to increased carcinogenicity of the affected liver [41]. Therefore, the link between skeletal muscle mass loss and tumor recurrence might be the byproduct of liver cirrhosis.

In the present meta-analysis, loss of skeletal muscle mass was defined by reviewing the transverse CT images obtained before the treatments for HCC or upon the diagnosis of HCC and estimating the muscle mass from outlining the cross-sections of multiple regions (the psoas, erector spinae, quadratus lumborum, transverse abdominis, external and internal oblique abdominal and rectus abdominis muscles). Compared with the diagnostic algorithm proposed by the European Working Group on Sarcopenia in Older People (EWGSOP) and Asian Working Group for Sarcopenia (AWGS) [42, 43], the method to define sarcopenia in our enrolled studies did not measure muscle strength or physical function. This is because most of the included studies employed a retrospective design and did not include both measurements at the data collection stage. A notable finding was that the diagnostic criteria for skeletal muscle mass loss varied across studies. We also found that with an increase in cut-off values of muscle mass (especially for male patients), there was an insignificant trend of stronger associations between skeletal muscle mass loss and all-cause mortality. This might reflect that the inclusion of patients with mild skeletal muscle mass loss (defined by a higher cut-off point) was even more strongly associated with poor survival. Therefore, a prospective study is warranted for identifying an appropriate threshold of sarcopenia for the prediction of mortality in HCC patients.

The mainstream treatment of skeletal muscle atrophy is exercise and protein supplement. Future studies of exercise and nutrition treatments on HCC and chronic liver disease are warranted since loss of skeletal muscle mass is the independent predictor of prognosis of HCC. In fact, there is some evidence showing that regular aerobic exercise decreased liver tumor development in a mice model [44], and branched-chain amino acid supplement was associated with less skeletal muscle atrophy in patients with HCC [45]. Besides, since most cases with HCC developed in patients with chronic liver disease, in which muscle volume loss 


\section{Liver Cancer}

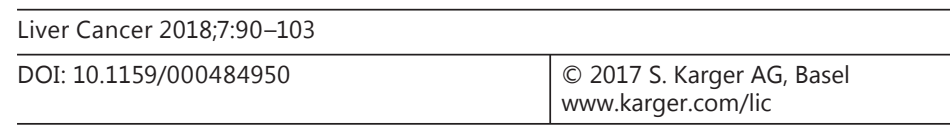

Chang et al.: Association between Skeletal Muscle Mass Loss and Hepatocellular Carcinoma

and muscle strength decrease, we should evaluate the muscle mass and strength and treat with exercise and nutritional supplement not only in patients with chronic liver disease but also in those with HCC [45].

There are several limitations to the current meta-analysis. First, the cancer stages of the included patients varied among studies. As the treatment types might partially reflect the disease severity (curative and palliative treatments for early and late stages, respectively), we performed a stratified analysis based on therapeutic approaches, which revealed no influence on the association between loss of skeletal muscle mass and mortality. However, the stratified analysis could not be conducted based on individual treatments because the outcomes of different treatment arms were not reported in each study. Second, because death or tumor recurrence usually occurs after some time, the event rates might be underestimated in the case of a short follow-up period. However, the follow-up durations varied among studies and were not defined in some of them, both of which might potentially influence our outcomes. Third, a potential publication bias was detected regarding the adjusted HRs of loss of skeletal muscle mass for overall survival. We speculated that it was derived from heterogeneity of the adjusted factors across the included studies, which led to asymmetrical distribution of estimated effects on the funnel plot. In addition, since there were various criteria to define sarcopenia, the prevalence of sarcopenia might vary in studies using different diagnostic protocols, leading to heterogeneity during the pooled analysis. Nevertheless, we believed the publication bias did not have a significant influence on our observation, because the statistical significance between both medical conditions remained after imputing unpublished researches into the funnel plot. Fourth, regarding grouping the cut-off values for skeletal muscle mass loss, there was an overlap of the ranges between group 2 and group 3 in female patients with HCC, which might explain why the trend of an increased association between loss of skeletal muscle mass and all-cause mortality was insignificant with the increase in the threshold values for skeletal muscle mass loss. Fifth, although liver cirrhosis might be a key factor to mediate overall survival and tumor recurrence between HCC and loss of skeletal muscle mass, not all the included studies specified the condition of liver cirrhosis in their patients. Therefore, we were not able to perform a stratified analysis based on the presence of liver cirrhosis in the present meta-analysis. Sixth, not all included studies reported overall and recurrence-free survival, although both outcomes are paramount in research in oncology. If the study lacked the data on recurrence-free survival, this might indicate a negative result regarding the association between tumor recurrence and the factor investigated. This could lead to another publication bias, and the finding should be carefully interpreted.

In conclusion, loss of skeletal muscle mass is associated with an increase in all-cause mortality and tumor recurrence in patients with HCC. The association between loss of skeletal muscle mass and survival is not modified by treatment options but might vary according to different cut-off points of muscle mass on transverse CT images. Considering the study limitations, further prospective studies incorporating measurements of muscles strength and physical function are warranted to determine whether inclusion of both parameters better predicts the outcome compared with the use of muscle mass only.

\section{Disclosure Statement}

The authors declare no conflicts of interest.

\section{Funding Sources}

The study was made possible by the research funding of the Community and Geriatric Medicine Research Center, National Taiwan University Hospital, Bei-Hu Branch. 


\section{References}

1 McGlynn KA, Petrick JL, London WT: Global epidemiology of hepatocellular carcinoma: an emphasis on demographic and regional variability. Clin Liver Dis 2015;19:223-238.

2 Altekruse SF, McGlynn KA, Reichman ME: Hepatocellular carcinoma incidence, mortality, and survival trends in the United States from 1975 to 2005. J Clin Oncol 2009;27:1485-1491.

3 Thein HH, Khoo E, Campitelli MA, Zaheen A, Yi Q, De P, Earle CC: Trends in relative survival in patients with a diagnosis of hepatocellular carcinoma in Ontario: a population-based retrospective cohort study. CMAJ Open 2015;3:E208-E216.

4 Xiao J, Li G, Lin S, He K, Lai H, Mo X, Chen J, Lin Y: Prognostic factors of hepatocellular carcinoma patients treated by transarterial chemoembolization. Int J Clin Exp Pathol 2014;7:1114-1123.

5 Colecchia A, Schiumerini R, Cucchetti A, Cescon M, Taddia M, Marasco G, Festi D: Prognostic factors for hepatocellular carcinoma recurrence. World J Gastroenterol 2014;20:5935-5950.

6 Han DS, Chang KV, Li CM, Lin YH, Kao TW, Tsai KS, Wang TG, Yang WS: Skeletal muscle mass adjusted by height correlated better with muscular functions than that adjusted by body weight in defining sarcopenia. Sci Rep 2016;6:19457.

7 Montero-Fernandez N, Serra-Rexach JA: Role of exercise on sarcopenia in the elderly. Eur J Phys Rehabil Med 2013;49:131-143.

8 Cruz-Jentoft AJ, Landi F, Schneider SM, Zuniga C, Arai H, Boirie Y, Chen LK, Fielding RA, Martin FC, Michel JP, Sieber C, Stout JR, Studenski SA, Vellas B, Woo J, Zamboni M, Cederholm T: Prevalence of and interventions for sarcopenia in ageing adults: a systematic review. Report of the International Sarcopenia Initiative (EWGSOP and IWGS). Age Ageing 2014;43:748-759.

9 Shachar SS, Williams GR, Muss HB, Nishijima TF: Prognostic value of sarcopenia in adults with solid tumours: a meta-analysis and systematic review. Eur J Cancer 2016;57:58-67.

10 Chang KV, Hsu TH, Wu WT, Huang KC, Han DS: Is sarcopenia associated with depression? A systematic review and meta-analysis of observational studies. Age Ageing 2017;46:738-746.

11 Chang KV, Hsu TH, Wu WT, Huang KC, Han DS: Association between sarcopenia and cognitive impairment: a systematic review and meta-analysis. J Am Med Dir Assoc 2016;17:1164e7-e1164e15.

12 Hung CY, Hsiao MY, Chang KV, Han DS, Wang TG: Comparative effectiveness of dextrose prolotherapy versus control injections and exercise in the management of osteoarthritis pain: a systematic review and metaanalysis. J Pain Res 2016;9:847-857.

13 Chang KV, Wu WT, Han DS, Ozcakar L: Ulnar nerve cross-sectional area for the diagnosis of cubital tunnel syndrome: a meta-analysis of ultrasonographic measurements. Arch Phys Med Rehabil, Epub ahead of print.

14 Wu WT, Chang KV, Han DS, Chang CH, Yang FS, Lin CP: Effectiveness of glenohumeral joint dilatation for treatment of frozen shoulder: a systematic review and meta-analysis of randomized controlled trials. Sci Rep 2017; 7:10507.

15 Tandon P, Ney M, Irwin I, Ma MM, Gramlich L, Bain VG, Esfandiari N, Baracos V, Montano-Loza AJ, Myers RP: Severe muscle depletion in patients on the liver transplant wait list: its prevalence and independent prognostic value. Liver Transpl 2012;18:1209-1216.

16 Harimoto N, Yoshizumi T, Shimokawa M, Sakata K, Kimura K, Itoh S, Ikegami T, Ikeda T, Shirabe K, Maehara Y: Sarcopenia is a poor prognostic factor following hepatic resection in patients aged 70 years and older with hepatocellular carcinoma. Hepatol Res 2016;46:1247-1255.

17 Nishikawa H, Nishijima N, Enomoto H, Sakamoto A, Nasu A, Komekado H, Nishimura T, Kita R, Kimura T, Iijima H, Nishiguchi S, Osaki Y: Prognostic significance of sarcopenia in patients with hepatocellular carcinoma undergoing sorafenib therapy. Oncol Lett 2017;14:1637-1647.

18 Kobayashi A, Kaido T, Hamaguchi Y, Okumura S, Taura K, Hatano E, Okajima H, Uemoto S: Impact of postoperative changes in sarcopenic factors on outcomes after hepatectomy for hepatocellular carcinoma. J Hepatobiliary Pancreat Sci 2016;23:57-64.

19 Mir O, Coriat R, Blanchet B, Durand JP, Boudou-Rouquette P, Michels J, Ropert S, Vidal M, Pol S, Chaussade S, Goldwasser F: Sarcopenia predicts early dose-limiting toxicities and pharmacokinetics of sorafenib in patients with hepatocellular carcinoma. PLoS One 2012;7:e37563.

20 Fujiwara N, Nakagawa H, Kudo Y, Tateishi R, Taguri M, Watadani T, Nakagomi R, Kondo M, Nakatsuka T, Minami T, Sato M, Uchino K, Enooku K, Kondo Y, Asaoka Y, Tanaka Y, Ohtomo K, Shiina S, Koike K: Sarcopenia, intramuscular fat deposition, and visceral adiposity independently predict the outcomes of hepatocellular carcinoma. J Hepatol 2015;63:131-140.

21 Harimoto N, Shirabe K, Yamashita YI, Ikegami T, Yoshizumi T, Soejima Y, Ikeda T, Maehara Y, Nishie A, Yamanaka T: Sarcopenia as a predictor of prognosis in patients following hepatectomy for hepatocellular carcinoma. Br J Surg 2013;100:1523-1530.

22 Itoh S, Shirabe K, Matsumoto Y, Yoshiya S, Muto J, Harimoto N, Yamashita Y, Ikegami T, Yoshizumi T, Nishie A, Maehara Y: Effect of body composition on outcomes after hepatic resection for hepatocellular carcinoma. Ann Surg Oncol 2014;21:3063-3068.

23 Kamachi S, Mizuta T, Otsuka T, Nakashita S, Ide Y, Miyoshi A, Kitahara K, Eguchi Y, Ozaki I, Anzai K: Sarcopenia is a risk factor for the recurrence of hepatocellular carcinoma after curative treatment. Hepatol Res 2016;46: 201-208. 
Chang et al.: Association between Skeletal Muscle Mass Loss and Hepatocellular Carcinoma

24 Levolger S, van Vledder MG, Muslem R, Koek M, Niessen WJ, de Man RA, de Bruin RW, Ijzermans JN: Sarcopenia impairs survival in patients with potentially curable hepatocellular carcinoma. J Surg Oncol 2015;112:208213.

25 Takagi K, Yagi T, Yoshida R, Shinoura S, Umeda Y, Nobuoka D, Kuise T, Watanabe N, Fujiwara T: Sarcopenia and American Society of Anesthesiologists physical status in the assessment of outcomes of hepatocellular carcinoma patients undergoing hepatectomy. Acta Med Okayama 2016;70:363-370.

26 Voron T, Tselikas L, Pietrasz D, Pigneur F, Laurent A, Compagnon P, Salloum C, Luciani A, Azoulay D: Sarcopenia impacts on short- and long-term results of hepatectomy for hepatocellular carcinoma. Ann Surg 2015; 261:1173-1183.

27 Yabusaki N, Fujii T, Yamada S, Suzuki K, Sugimoto H, Kanda M, Nakayama G, Koike M, Fujiwara M, Kodera Y: Adverse impact of low skeletal muscle index on the prognosis of hepatocellular carcinoma after hepatic resection. Int J Surg 2016;30:136-142.

28 Iritani S, Imai K, Takai K, Hanai T, Ideta T, Miyazaki T, Suetsugu A, Shiraki M, Shimizu M, Moriwaki H: Skeletal muscle depletion is an independent prognostic factor for hepatocellular carcinoma. J Gastroenterol 2015;50: 323-332.

29 Meza-Junco J, Montano-Loza AJ, Baracos VE, Prado CM, Bain VG, Beaumont C, Esfandiari N, Lieffers JR, Sawyer MB: Sarcopenia as a prognostic index of nutritional status in concurrent cirrhosis and hepatocellular carcinoma. J Clin Gastroenterol 2013;47:861-870.

30 Valero V, 3rd, Amini N, Spolverato G, Weiss MJ, Hirose K, Dagher NN, Wolfgang CL, Cameron AA, Philosophe B, Kamel IR, Pawlik TM: Sarcopenia adversely impacts postoperative complications following resection or transplantation in patients with primary liver tumors. J Gastrointest Surg 2015;19:272-281.

31 Dodson RM, Firoozmand A, Hyder O, Tacher V, Cosgrove DP, Bhagat N, Herman JM, Wolfgang CL, Geschwind JF, Kamel IR, Pawlik TM: Impact of sarcopenia on outcomes following intra-arterial therapy of hepatic malignancies. J Gastrointest Surg 2013;17:2123-2132.

32 Hiraoka A, Hirooka M, Koizumi Y, Izumoto H, Ueki H, Kaneto M, Kitahata S, Aibiki T, Tomida H, Miyamoto Y, Yamago H, Suga Y, Iwasaki R, Mori K, Miyata H, Tsubouchi E, Kishida M, Ninomiya T, Abe M, Matsuura B, Kawasaki H, Hiasa Y, Michitaka K: Muscle volume loss as a prognostic marker in hepatocellular carcinoma patients treated with sorafenib. Hepatol Res 2017;47:558-565.

33 Woo N, Kim SH: Sarcopenia influences fall-related injuries in community-dwelling older adults. Geriatr Nurs 2014;35:279-282.

34 Martinez BP, Batista AK, Gomes IB, Olivieri FM, Camelier FW, Camelier AA: Frequency of sarcopenia and associated factors among hospitalized elderly patients. BMC Musculoskelet Disord 2015;16:108.

35 Chang SF, Lin PL: Systematic literature review and meta-analysis of the association of sarcopenia with mortality. Worldviews Evid Based Nurs 2016;13:153-162.

36 Peake JM, Della Gatta P, Suzuki K, Nieman DC: Cytokine expression and secretion by skeletal muscle cells: regulatory mechanisms and exercise effects. Exerc Immunol Rev 2015;21:8-25.

37 Elmashad N, Ibrahim WS, Mayah WW, Farouk M, Ali LA, Taha A, Elmashad W: Predictive value of serum insulin-like growth factor-1 in hepatocellular carcinoma. Asian Pac J Cancer Prev 2015;16:613-619.

38 Sachdeva M, Chawla YK, Arora SK: Immunology of hepatocellular carcinoma. World J Hepatol 2015;7:20802090.

39 Argiles JM, Busquets S, Stemmler B, Lopez-Soriano FJ: Cachexia and sarcopenia: mechanisms and potential targets for intervention. Curr Opin Pharmacol 2015;22:100-106.

40 Montano-Loza AJ: New concepts in liver cirrhosis: clinical significance of sarcopenia in cirrhotic patients. Minerva Gastroenterol Dietol 2013;59:173-186.

41 Yamamoto Y, Ikoma H, Morimura R, Konishi H, Murayama Y, Komatsu S, Shiozaki A, Kuriu Y, Kubota T, Nakanishi M, Ichikawa D, Fujiwara H, Okamoto K, Sakakura C, Ochiai T, Otsuji E: Optimal duration of the early and late recurrence of hepatocellular carcinoma after hepatectomy. World J Gastroenterol 2015;21:12071215.

42 Patel HP, Syddall HE, Jameson K, Robinson S, Denison H, Roberts HC, Edwards M, Dennison E, Cooper C, Aihie Sayer A: Prevalence of sarcopenia in community-dwelling older people in the UK using the European Working Group on Sarcopenia in Older People (EWGSOP) definition: findings from the Hertfordshire Cohort Study (HCS). Age Ageing 2013;42:378-384.

43 Chen LK, Lee WJ, Peng LN, Liu LK, Arai H, Akishita M; Asian Working Group for Sarcopenia: Recent advances in sarcopenia research in Asia: 2016 update from the Asian Working Group for Sarcopenia. J Am Med Dir Assoc 2016;17:767 e761-e767.

44 Piguet AC, Saran U, Simillion C, Keller I, Terracciano L, Reeves HL, Dufour JF: Regular exercise decreases liver tumors development in hepatocyte-specific PTEN-deficient mice independently of steatosis. J Hepatol 2015; 62:1296-1303.

45 Koya S, Kawaguchi T, Hashida R, Goto E, Matsuse H, Saito H, Hirota K, Taira R, Matsushita Y, Imanaga M, Nagamatsu A, Shirono T, Shimose S, Iwamoto H, Niizeki T, Kuromatsu R, Miura H, Shiba N, Torimura T: Effects of in-hospital exercise on liver function, physical ability, and muscle mass during treatment of hepatoma in patients with chronic liver disease. Hepatol Res 2017;47:E22-E34. 